\section{Endoscopic ultrasound-guided tattooing of a retroesophageal parathyroid adenoma}

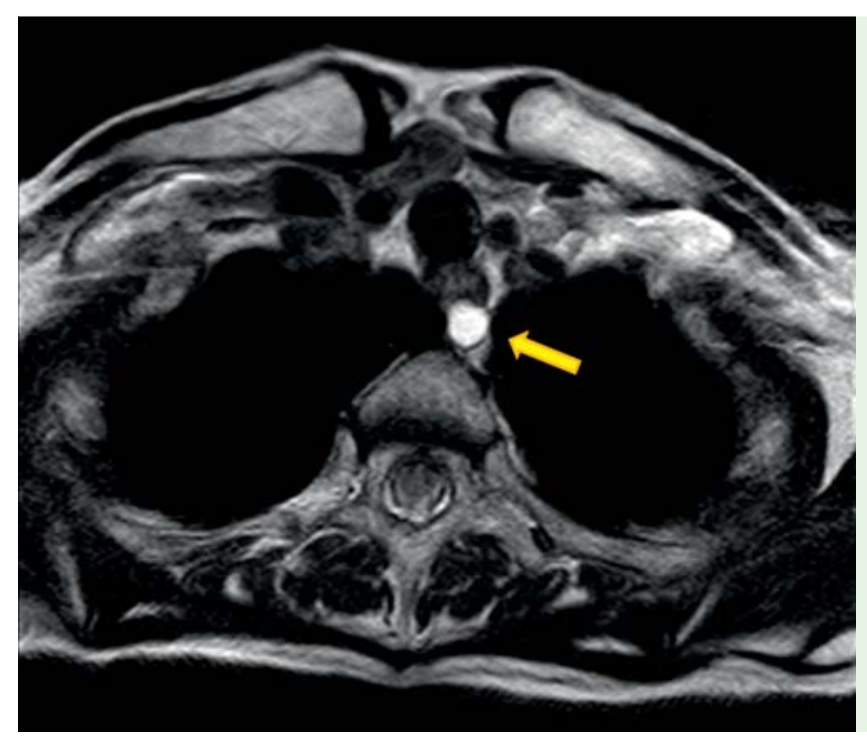

Fig. 1 Magnetic resonance imaging showed a small retroesophageal mass (arrow) at the level of the first dorsal vertebral body.


Fig. 2 11C-methionine positron emission tomography-computed tomography images demonstrated a focal area of uptake in the retroesophageal space (arrows). This finding was suspicious for ectopic enlarged parathyroid gland.
Primary hyperthyroidism (PHPT) results from an ectopic adenoma located in a retroesophageal space in about $3 \%$ of cases [1]. In these patients, parathyroidectomy is a challenge even for skilled surgeons due to difficulty in identifying the lesion in a very limited operating space between the cervical and thoracic regions. Preoperative imaging techniques include ultrasound, positron emission tomography-computed tomography (PET-CT) and magnetic resonance imaging (MRI) $[2,3]$. Endoscopic ultrasound (EUS) has been proposed as a tool for detecting parathyroid adenomas [4-6]. This report describes the case of a 74-year-old woman with PHPT.

Preoperative 99mTc-sestamibi scintigraphy showed a parathyroid adenoma behind the esophagus, which was confirmed by MRI ( Fig.1) and 11C-methionine PET-CT ( $\bullet$ Fig. 2). In an attempt to facilitate surgical identification, an operative EUS was performed in order to tattoo the target nodule.

The adenoma was visible from the upper esophagus as a 13-mm iso-hyperechoic nodule with a peripheral cyst, behind the esophagus. A 25-gauge needle (EchoTip Ultra; Cook Medical, Limerick, Ireland) was used under EUS guidance to inject $1 \mathrm{~mL}$ of ink into the nodule ( $\bullet$ Fig. 3 ). During the injection, a hyperechoic blush was visualized around the tip of the needle. No complications were observed.

The patient underwent surgery 3 days after the tattooing procedure. A videoassisted parathyroidectomy was performed. Behind the esophagus, the black tattooed nodule guided the surgeons to perform a gentle dissection and excision of the adenoma. The tattoo was extremely precise and no ink had spread into the surrounding tissue ( $\bullet$ Fig. 4 ). The intraoperative serum parathyroid hormone assay showed a drop to the normal range, and calcium serum level reached the normal value within the first postoperative day. EUS-guided tattooing has been used to mark the location of small pancreatic neuroendocrine tumors [7]. The present case represents the first report of EUS-guided tattooing of a retroesophageal parathyroid adenoma. Its use for preoperative marking of a small tumor can be helpful to the surgeon by making surgical removal more precise and less invasive and thus avoiding unnecessary dissection. 


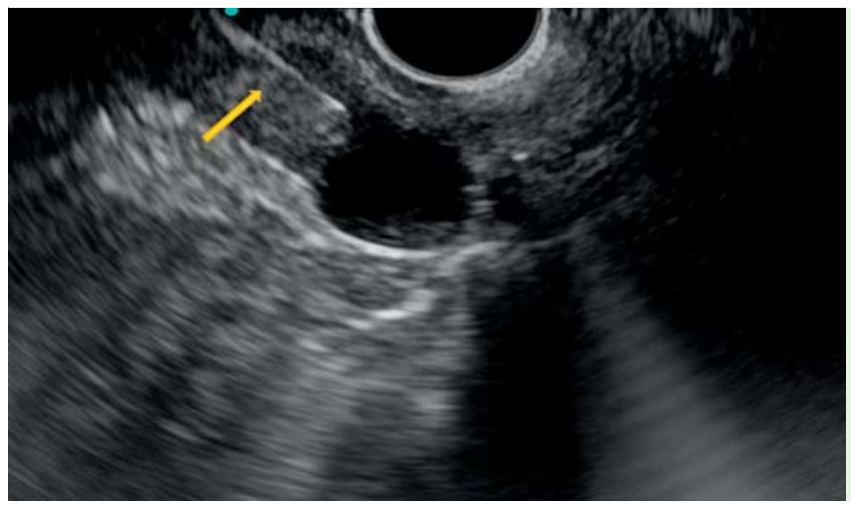

Fig. 3 Endoscopic ultrasound-guided fineneedle injection of ink into the parathyroid adenoma. The 25 -gauge needle was seen as a hyperechoic line (arrow) $1 \mathrm{~mL}$ of sterile purified ink (dilution 0.5:10000) was injected into the nodule and it was seen as a hyperechoic cloud inside the adenoma.
Endoscopy_UCTN_Code_TTT_1AS_2AG

\section{Competing interests: None}

\section{Silvia Carrara', Luca Cozzaglio², Manol Jovani ${ }^{1}$, Giovanna Pepe ${ }^{3}$, Cristiana Bonifacio ${ }^{4}$, Andrea Ander- Ioni $^{1}$, Alessandro Repici ${ }^{1}$}

${ }^{1}$ Digestive Endoscopy Unit, Istituto Clinico Humanitas, Rozzano, Milan, Italy

${ }^{2}$ Division of Surgical Oncology, Istituto Clinico Humanitas, Rozzano, Milan, Italy

${ }^{3}$ Nuclear Medicine, Istituto Clinico Humanitas, Rozzano, Milan, Italy

${ }^{4}$ Radiology, Istituto Clinico Humanitas, Rozzano, Milan, Italy

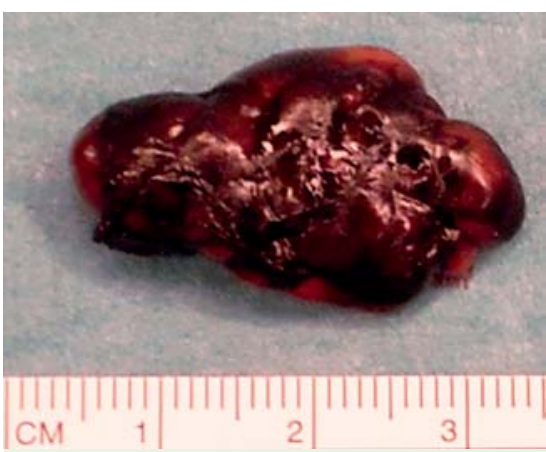

Fig. 4 The black tattooed nodule guided the surgeons to perform a gentle excision of the adenoma. Pathological analysis confirmed parathyroid adenoma.

with real-time rapid parathyroid hormone assay. J Am Coll Surg 2009; 209: e1 - 4

7 Lennon AM, Newman N, Makary MA et al. EUS-guided tattooing before laparoscopic distal pancreatic resection. Gastrointest Endosc 2010; 72: 1089-1094

2 Purz S, Kluge R, Barthel $H$ et al. Visualization of ectopic parathyroid adenomas. N Engl J Med 2013; 369: 2067-2069

3 Udelsman R, Pasieka JL, Sturgeon C et al. Surgery for asymptomatic primary hyperparathyroidism: proceedings of the third international workshop. J Clin Endocrinol Metab 2009; 94: 366-372

4 Ersoy R, Ersoy O, Evranos Ogmen B et al. Diagnostic value of endoscopic ultrasonography for preoperative localization of parathyroid adenomas. Endocrine. In press 2014. doi: 10.1007/s12020-013-0152-3

5 Fusaroli P, Kypraios D, Caletti G et al. Pancreatico-biliary endoscopic ultrasound: a systematic review of the levels of evidence, performance and outcomes. World J Gastroenterol 2012; 18: $4243-4256$

6 Graff-Baker A, Roman SA, Boffa D et al. Diagnosis of ectopic middle mediastinal parathyroid adenoma using endoscopic ultrasonography-guided fine-needle aspiration 